\title{
How different is the dietary intake of individuals with metabolic syndrome?
}

\begin{abstract}
Background: Metabolic syndrome has turned out to be a chief public health concern, but the role of diet in the etiology of metabolic syndrome is not well understood. Aims: This study aimed at assessing whether individuals with metabolic syndrome had a specific dietary intake and how it compared with the Recommended Nutrient Intakes for Malaysia and the Medical Nutrition Therapy Guidelines by the Malaysian Dietitians' Association. Study Design: Crosssectional study. Place of Study: Putrajaya, Malaysia. Duration of Study: Eight weeks. Methodology: Waist circumference, systolic blood pressure, diastolic blood pressure, triglycerides, HDL cholesterol and fasting plasma glucose were collected in 659 randomly selected subjects. A total of 275 out of 322 subjects with metabolic syndrome completed the self-administered three-day food record. Data were analysed with Nutritionist Pro, and were compared with the Recommended Nutrient Intakes for Malaysia (2010), based on age and gender. Metabolic syndrome was defined according to the 'Harmonized' criteria. Results: Subjects' mean age was 34.51 \pm 8.30 years, 202 Females: 73 Males. Comparison of subjects' food records with the Recommended Nutrient Intakes for Malaysia revealed that protein consumption was significantly higher among study subjects compared to the Recommended Nutrient Intakes for Malaysia (62 g/day for males; $55 \mathrm{~g} /$ day for females), $P<.001$. All subjects $(100 \%)$ had low fibre ( $<20 \mathrm{~g} /$ day) intake. Conversely, most subjects $(72.0 \%)$ had high sodium intake ( $\geq 2400 \mathrm{mg} /$ day). Conclusion: This study highlights the importance of assessing the dietary intake of each individual with metabolic syndrome. There is a discrepancy between the dietary intake among individuals with metabolic syndrome and the Recommended Nutrient Intakes for Malaysia or Medical Nutrition Therapy Guidelines by the Malaysian Dietitians' Association. Notwithstanding, our study cannot declare that diet induces the disease, yet it definitely sheds more light on the need for appropriate and professional dietary measures to be integrated in the management of individuals with metabolic syndrome.
\end{abstract}

Keyword: Metabolic syndrome; Dietary intake; Medical nutrition therapy; Recommended nutrient intakes 